\title{
Erratum: Multipartite-entanglement monotones and polynomial invariants [Phys. Rev. A 85, 022301 (2012)]
}

Christopher Eltschka, Thierry Bastin, Andreas Osterloh, and Jens Siewert

(Received 9 May 2012; published 16 May 2012)

DOI: 10.1103/PhysRevA.85.059903

PACS number(s): 03.67.Mn, 99.10.Cd

The two-column equation following Eq. (2) of our article is incorrect and, therefore, the proof of Theorem 1 is not complete. However, we emphasize that the theorem is valid. In the following we present a correct proof.

We first note that by factoring out $a b$ in the first term in Eq. (2) and $\sqrt{\left(1-a^{2}\right)\left(1-b^{2}\right)}$ in the second term, the inequality can be written as

$$
f_{\eta}(a, b, x)+f_{\eta}\left(\sqrt{1-a^{2}}, \sqrt{1-b^{2}}, x\right) \leqslant 1,
$$

where

$$
f_{\eta}(\alpha, \beta, x)=\alpha \beta\left[\frac{\alpha \beta}{x \alpha^{2}+(1-x) \beta^{2}}\right]^{\frac{\eta}{2}-1} .
$$

Now for $a, b \neq 0,1$, for both terms the base of the exponential in $f_{\eta}(\alpha, \beta, x)$ is positive. Since the exponential function for positive bases is always convex, it follows that

$$
f_{\eta}(\alpha, \beta, x) \leqslant\left(1-\frac{\eta}{4}\right) f_{0}(\alpha, \beta, x)+\frac{\eta}{4} f_{4}(\alpha, \beta, x) .
$$

Therefore, if Eq. (2) is true for both $\eta=0$ and $\eta=4$, it holds also for all values $0<\eta<4$. For $\eta=0$, a straightforward calculation shows that the sum in Eq. (2) gives exactly 1 , and for $\eta=4$, the inequality was proved by Wong and Christensen in Ref. [1], which concludes our proof for $a, b \neq 0,1$.

In order to treat the cases where one of the parameters $a$ or $b$ equals 0 or 1 , we note that $f_{\eta}(\alpha, \beta, x)$ continuously goes to zero if only one of $\alpha$ or $\beta$ goes to zero (and, of course, is also continuous at $\alpha=1$ or $\beta=1$ ). Therefore the inequality still holds in this limit. Note that this also covers the cases $a=0, b=1$ and $a=1, b=0$.

The only remaining cases are $a=b=0$ and $a=b=1$ so that Eq. (2) is not well defined. But then the POVM reduces to a unitary transformation for which the function $\mu$ is constant by definition.

We thank S. Szalay for pointing out the error in our previous argument.

[1] A. Wong and N. Christensen, Phys. Rev. A 63, 044301 (2001). 